\title{
A Novel Sequential Circuit Optimization with Clock Gating Logic
}

\author{
Yu-Min Kuo Shih-Hung Weng Shih-Chieh Chang \\ Department of CS, National Tsing Hua University, Hsinchu, Taiwan \\ \{ymkuo, shweng, scchang\}@cs.nthu.edu.tw
}

\begin{abstract}
-
To save power consumption, it has been shown that the clock signal can be gated without changing the functionality under certain clock-gating conditions. We observe that the clock-gating conditions and the next-state function of a Flip-Flop (FF) are correlated and can be used for sequential optimization. We show that the implementation of the next-state function of any FF can be just an inverter if the clock signal is appropriately gated. By exploiting the flexibility between the clock-gating conditions and the nextstate function, we propose an iterative optimization technique to minimize the overall timing.
\end{abstract}

\section{Introduction}

A sequential circuit consists of combinational elements to compute next states and sequential elements such as Flip-Flops (FF) to store the current states. When a clock pulse arrives, a circuit re-evaluates the states. Normally, clock signals are delivered to all FFs periodically; however, it has been shown that it is not necessary to deliver a clock pulse to an FF in every clock cycle. Techniques such as clock gating [1][2][3][4][5][6][7][8][9][10][11][12] shut off clock signals when a circuit is in idle state or when FFs need not change their states so as to save the power consumption.

In this paper, we propose novel flexibility for sequential optimization using the concept of clock gating, the novel flexibility of which is completely different from traditional sequential don't cares. The new structure is shown in Figure 1 where the clock of an FF is gated when the clock-gating function is asserted and the next-state function provides the next-state value of the FF. In addition, a $C G$ Cell consisting of a latch and an $A N D$ gate is used to avoid glitches. There should also be logic circuits computing the primary outputs which are omiited in the figure.

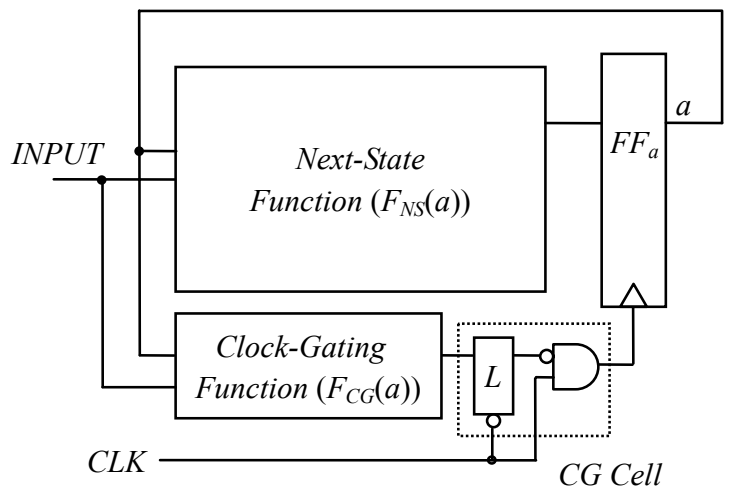

Figure 1: Basic Structure of a Sequential Circuit with the Clock Gating

\begin{tabular}{|c|c|c|c|c|c|}
\hline \multicolumn{3}{|c|}{ Current State } & \multicolumn{3}{|c|}{ Next State } \\
\hline$a$ & $b$ & $c$ & $a$ & $b$ & $c$ \\
\hline$\theta$ & 0 & 0 & $\theta$ & 0 & 1 \\
\hline$\theta$ & 0 & 1 & $\theta$ & 1 & 0 \\
\hline$\theta$ & 1 & 0 & $\theta$ & 1 & 1 \\
\hline 0 & 1 & 1 & 1 & 0 & 0 \\
\hline 1 & 0 & 0 & 1. & 0 & 1 \\
\hline 1. & 0 & 1 & 1. & 1 & 0 \\
\hline 1 . & 1 & 0 & 1. & 1 & 1 \\
\hline 1 & 1 & 1 & 0 & 0 & 0 \\
\hline
\end{tabular}

Figure 2: State Transition Table of a 3-Bit Counter

We illustrate how the new architecture works by a 3-bit counter. Figure 2 shows the state transition table of a 3-bit counter where $a$ is the $3^{\text {rd }}$ bit and $F F_{a}$ is the corresponding storage element. When the current state $(a, b, c)$ is $(0,0,0)$, the next state will be $(0,0,1)$ and the state of $F F_{a}$ will not change its value. According to the state transition table in Figure 2, when the current state $(a, b, c)$ is equal to one of states in $S=\{(0,0,0),(0,0,1),(0,1,0),(1,0,0),(1,0,1)$, $(1,1,0)\}$, the state of $F F_{a}$ does not change. Since $F F_{a}$ does not change its value for those current states in $S$, a clock 
pulse dose not need to arrive at $F F_{a}$ and can be gated. We can derive a Boolean function $b^{\prime}+c^{\prime}$ to characterize current states in $S$. Since no clock pulse is delivered to $F F_{a}$ when the condition $b^{\prime}+c^{\prime}$ is true, we can randomly assign the output of the next-state function for those conditions; in other words, we can use $b^{\prime}+c^{\prime}$ as the don't-care function to minimize the original next-state function $a b^{\prime}+a c^{\prime}+a^{\prime} b c$. The result of minimization is an inverter, $a^{\prime}$.

Still, the Boolean conditions to determine when there should be a clock pulse can be complicated for some sequential circuits. In this paper, we present theoretical foundations and efficient heuristics for building sequential circuits consisting of the clock-gating functions and the next-state functions. Conceptually, our algorithm transforms some combinational logics to the clock-gating function. And in many cases, the transformation can improve the overall efficiency of a circuit. We have performed our experiments on a set of benchmark circuits and obained on the average $13.99 \%$ timing improvement in TSMC $0.13 \mu \mathrm{m}$ library.

The remainder of this paper is organized as follows. Section 2 shows the overall algorithm and the process flow. Section 3 presents the experimental results. Section 4 concludes this paper.

\section{Logic Synthesis Using the Clock Gating Function}

\subsection{Basic definitions and key facts}

We first present two simple but very important facts about the relationship between the clock-gating function and the next-state function for a single FF. The facts form the foundations of all following equations and heuristics. Note that the clock of an FF is shut off when the clock-gating function is asserted in Figure 1.

FACT1: When the clock of an FF is gated, the next state of the FF remains the same regardless of whether the next-state function is zero or one. Therefore, the on-set of the clock-gating function can be the don't-care set for the next-state function [13].

FACT2: When the next state and the current state value of the FF are the same, the FF remains its state value regardless of whether the clock is gated or not. Therefore, the conditions when the next- state value is equal to the current state of the FF can be the don't-care set for the clock-gating function.

In the following, we use $F_{C G}$ to denote the clock-gating function and use $F_{N S}$ to denote the next-state function. Let us consider $F F_{a}$ in Figure 1 where $F_{N S}(a)$ is the next-state function and $F_{C G}(a)$ is the clock-gating function of $F F_{a}$. In addition, signal $a$ is the output of $F F_{a}$ as well as an input of the $F_{N S}(a)$ and $F_{C G}(a)$. To describe the facts precisely, we present them in mathematical form.

FACT1: When $F_{C G}(a)=1, F_{N S}(a)$ can be 0 or 1 . Thus, the on-set of $F_{C G}(a)$ is the don't-care set for $F_{N S}(a)$.

FACT2: When $\left(a \equiv F_{N S}(a)\right)=1, F_{C G}(a)$ can be 0 or 1 . Thus, the on-set of $\left(a \equiv F_{N S}(a)\right)$ is the don't-care set for $F_{C G}(a)$ where the symbol " $\equiv$ " represents the Boolean operator XNOR.

\subsection{The simplest implementation of $F_{N S}$ and $F_{C G}$}

We can use don't-care conditions in FACT1 and FACT2 to minimize $F_{N S}$ and $F_{C G}$ respectively. In the following, we discuss a very efficient implementation for $F_{N S}$ and $F_{C G}$. To distinguish between the original next-state function and the newly generated next-state function, we use $F_{O R I-N S}$ to denote the original implementation of the next-state function without the clock gating.

Without going into a complicated proof, it is easy to show that the simplest implementation of $F_{C G}$ is that $F_{C G}=$ 0 , because there exists a legal solution that the clock is not gated at all. When $F_{C G}=0$, according to FACT1, there is no don't care for $F_{N S}$, so that $F_{N S}=F_{O R I-N S}$.

Now, we are interested in finding the simplest implementation of $F_{N S}$. The following theorem shows that $F_{N S}(a)$ after the optimization is a simple literal $a^{\prime}$.

Theorem 1: Let the on-set be $F_{\text {ORI-NS }}(a)$ and the don't-care set be $\left(a \equiv F_{\text {ORI-NS }}(a)\right)$. There exists a don't-care assignment such that the implementation of $F_{N S}(a)$ is $a^{\prime}$.

Proof: Omitted.

We have shown that the simplest implementation for $F_{C G}$ is 0 and an implementation for $F_{N S}$ can be just an inverter. However, if the simplest implementation for one of them is chosen, there will be no flexibility for the other 
function.

\subsection{Heuristic minimization for $F_{N S}$ and $F_{C G}$}

In the traditional design flow, we always choose the simplest implementation of $F_{C G}=0$. Therefore, the nextstate function $F_{\text {ORI-NS }}(a)$ does not have any don't cares. In this section, we would like to explore other alternatives of implementations for $F_{N S}$ and $F_{C G}$. According to FACT1 and FACT2, both $F_{N S}$ and $F_{C G}$ are correlated. Choosing one implementation may affect the don't-care set of the other.

Our idea is to use an iterative approach to simplify both functions. Before describing our iterative procedure, we rewrite FACT1 and FACT2 by the following two equations.

$$
\begin{aligned}
& F_{N S} *(a)<=\left\{\text { on-set }=F_{N S}(a), \text { dc-set }=F_{C G}(a)\right\} \\
& F_{C G} *(a)<=\left\{\text { on-set }=F_{C G}(a), \text { dc-set }=\left(a \equiv F_{N S}(a)\right)\right\}
\end{aligned}
$$

where $F_{N S}^{*}(a)$ is the simplified next-state function and $F_{C G} *(a)$ is the simplified clock-gating function.

Our iterative optimization requires initial Boolean functions of $F_{N S}$ and $F_{C G}$. Since we have the initial nextstate function $F_{N S}=F_{\text {ORI-NS }}(a)$, we need to find an appropriate initial clock-gating function $F_{C G}=F_{I N I-C G}$. How $F_{I N I-C G}$ is determined will be described later. Let us assume $F_{I N I-C G}$ is available. According to $\mathrm{EQ}(1)$, we can use $F_{N S}=$ $F_{O R I-N S}$ as the on-set and $F_{C G}=F_{I N I-C G}$ as the don't-care set to obtain a simplified $F_{N S} *$. In other words,

$$
F_{N S} *(a)<=\left\{\text { on-set }=F_{\text {ORI-NS }}(a), \text { dc-set }=F_{I N I-C G}(a)\right\} .
$$

Once the simplified next-state function $F_{N S} *$ is determined, according to $\mathrm{EQ}(2)$, we can use $F_{C G}=F_{I N I-C G}$ as the on-set and $\left(a \equiv F_{N S} *(a)\right)$ as the don't-care set to obtain simplified $F_{C G}$.

$$
F_{C G} *(a)<=\left\{\text { on-set }=F_{I N I-C G}(a), \text { dc-set }=\left(a \equiv F_{N S} *(a)\right)\right\} .
$$

After that, we can assign $F_{C G}=F_{C G} *$ and $F_{N S}=F_{N S} *$ and then use $\mathrm{EQ}(1)$ and $\mathrm{EQ}(2)$ to iteratively simplify both functions again.

The above iterative process requires an initial clockgating function. We now describe our selection for the initial function of $F_{C G}$ called $F_{I N I-C G}$. In our heuristic, we choose either of the following two Boolean functions.

$$
\begin{aligned}
& F_{\text {INI-CG }}(a)=a^{\prime} *\left(\left.\left(a \equiv F_{\text {ORI-NS }}(a)\right)\right|_{a=0}\right) . \\
& F_{I N I-C G}(a)=a *\left(\left.\left(a \equiv F_{\text {ORI-NS }}(a)\right)\right|_{a=1}\right) .
\end{aligned}
$$

where the symbol "|" denotes the cofactor operator. In our heuristic, if the number of fanouts of variable $a^{\prime}$ in $F_{N S}$ is larger than the number of fanouts of variable $a$, we choose $\mathrm{EQ}(3)$; otherwise, we choose $\mathrm{EQ}(4)$. It is because $\mathrm{EQ}(3)$ has better chance to minimize equations containing $a^{\prime}$ while $\mathrm{EQ}(4)$ is better for $a$.

The iterative heuristic method can be extended to simplify a whole circuit for the timing optimization. First, we perform a trial run of delay optimization to obtain the critical FFs whose inputs or outputs are in the "long" paths. The long paths can be defined as those paths whose path delay is less than $20 \%$ of the delay of a longest path. We then choose several FFs that are the end points of the critical paths and then apply our iterative heuristic method to these FFs for at most $k$ times of iteration loops where $k$ is chosen to be 5 in our experiments.

\section{Experimental Results}

We implemented the method in Section 2.3 and applied it to iscas89 sequential benchmark circuits. We use TSMC $0.13 \mu \mathrm{m}$ library as the technology libraries and Synopsys Design Compiler ${ }^{\circledR}$ for timing optimization. Table 1 shows the results of TSMC library. Columns 1 and 2 show the name and the number of FFs of a benchmark circuit. Column 3 shows the longest delay after using SIS script.delay. Column 4 shows the longest delay of the whole circuit. Column 5 shows the longest delay of only the next-state functions $F_{N S}$, and column 6 shows the longest delay of only the clock-gating function $F_{C G}$. In addition to $F_{N S}$ and $F_{C G}$, our resulting circuit also consists of logics computing primary outputs. Therefore, the longest delay of the whole circuit in column 4 may be larger than the longest delay of $F_{N S}$ and $F_{C G}$. Column 7 shows the ratio of timing improvement. Columns 8 and 9 show the area results before and after timing optimization, respectively. Column 10 shows the ratio of area penalty. The run time of our algorithm is shown in the last column. On average, the timing improvement is about $13.99 \%$ in the TSMC library. In addition, a circuit also consists of logic to compute primary outputs. If critical paths of a circuit are located in the paths to primary outputs, our timing optimization technique will not be efficient. It is because our technique 
Table 1: Results using Synopsys Design Compiler ${ }^{\circledR}$ with TSMC $0.13 \mu \mathrm{m}$ library

\begin{tabular}{|c|c|c|c|c|c|c|c|c|c|c|}
\hline \multirow{3}{*}{ Circuit } & \multirow{3}{*}{ \# FFs } & \multicolumn{5}{|c|}{ Timing (ns) } & \multicolumn{3}{|c|}{ Area $\left(\mu \mathrm{m}^{2}\right)$} & \multirow{3}{*}{$\begin{array}{c}\text { Runtime } \\
\text { (sec.) }\end{array}$} \\
\hline & & \multirow{2}{*}{ Original } & \multicolumn{3}{|c|}{ Optimized } & \multirow{2}{*}{$\begin{array}{c}\text { Improvement } \\
(\%)\end{array}$} & \multirow{2}{*}{ Original } & \multirow{2}{*}{ Optimized } & \multirow{2}{*}{$\begin{array}{c}\text { Overhead } \\
(\%)\end{array}$} & \\
\hline & & & Whole & $F_{N S}$ & $F_{C G}$ & & & & & \\
\hline s27 & 3 & 0.11 & 0.10 & 0.10 & 0.10 & 9.09 & 156.16 & 151.07 & -3.26 & 7.1 \\
\hline $\mathrm{s} 820$ & 5 & 0.88 & 0.66 & 0.63 & 0.61 & 25.00 & 4877.13 & 5302.35 & 8.73 & 58.0 \\
\hline $\mathrm{s} 832$ & 5 & 0.89 & 0.69 & 0.63 & 0.61 & 22.47 & 5103.24 & 5229.24 & 2.48 & 57.9 \\
\hline s1494 & 6 & 1.38 & 1.22 & 1.19 & 1.01 & 11.59 & 3829.33 & 4114.50 & 7.45 & 22.3 \\
\hline s510 & 6 & 0.75 & 0.67 & 0.46 & 0.67 & 10.67 & 3951.76 & 3805.40 & -3.70 & 47.9 \\
\hline s208 & 8 & 0.61 & 0.55 & 0.02 & 0.25 & 9.84 & 1111.02 & 758.42 & -31.74 & 37.2 \\
\hline $\mathrm{s} 344$ & 15 & 1.17 & 0.91 & 0.89 & 0.83 & 22.22 & 909.81 & 1351.13 & 48.51 & 60.9 \\
\hline s349 & 15 & 1.14 & 0.90 & 0.89 & 0.78 & 21.05 & 906.41 & 1378.29 & 52.06 & 71.3 \\
\hline s641 & 19 & 0.83 & 0.75 & 0.67 & 0.59 & 9.64 & 6879.00 & 7770.47 & 12.96 & 89.1 \\
\hline s526 & 21 & 0.59 & 0.53 & 0.48 & 0.42 & 10.17 & 3206.65 & 2780.87 & -13.28 & 95.3 \\
\hline s526n & 21 & 0.59 & 0.53 & 0.48 & 0.42 & 10.17 & 3206.65 & 2780.87 & -13.28 & 95.2 \\
\hline s1423 & 71 & 3.61 & 3.02 & 2.68 & 2.18 & 16.34 & 3846.31 & 4056.79 & 5.47 & 1341.3 \\
\hline s9234 & 211 & 1.94 & 1.87 & 1.83 & 1.73 & 3.61 & 11124.76 & 12092.28 & 8.70 & 961.3 \\
\hline AVG & & & & & & 13.99 & & & 6.24 & \\
\hline
\end{tabular}

can only reduce the delay to FFs.

\section{Conclusions}

In this paper, we first propose the flexibility provided by the concept of the clock gating. Then, we present the theoretical facts and efficient heuristics to optimize a sequential circuit consisting of the clock-gating functions and the next-state functions. On average, the timing of sequential benchmark circuits can be reduced about $13.99 \%$ in the TSMC library.

\section{REFERENCES}

[1] P. Babighian, L. Benini, and E. Macii, "A Scalable Algorithm for RTL Insertion of Gated Clocks Based on ODCs Computation," IEEE Trans. on CAD, vol. 24, no. 1, Jan 2005.

[2] M. Alidina, J. Monteiro, S. Devadas, and A. Ghosh, "Precomputation-Based Sequential Logic Optimization for Low Power," Proc. of ICCAD, pp. 74-81, 1994.

[3] L. Benini, and G. De Micheli, "Automatic Synthesis of Low-Power Gated-Clock Finite-State Machines," IEEE Trans. on CAD, vol. 15, no. 6, Jun. 1996.

[4] M. Müch, B. Wurth, R. Mehra, J. Sproch, and N. When, "Automating RT-Level Operand Isolation to Minimize Power Consumption in Datapaths," Proc. of DATE, pp. 624-633, 2000.

[5] V. Tiwari, S. Malik, and P. Ashar, "Guarded Evaluation: Pushing Power Management to Logic Synthesis/Design," Proc. of ISPLED, pp. 221-226, 1995.
[6] H. Kapadia, L. Benini, and G. De Micheli, "Reducing Switching Activity on Datapath Buses with Control-Signal Gating," IEEE J. of Solid-State Circuits, vol. 34, no. 3, March 1999.

[7] M. Onishi, A. Yamada, H. Noda, and T. Kambe, "A Method of Redundant Clocking Detection and Power Reduction at RT Level Design," Proc. of ISLPED, pp. 131-136, 1997.

[8] L. Benini, G. De Micheli, E. Macii, M. Poncino, and R. Scarsi, "Symbolic Synthesis of Clock-Gating Logic for Power Optimization of Synchronous Controllers," ACM Trans. on Design Automation Electronic Systems, vol. 4, no. 4, pp. 351-375, 1999.

[9] G. Lakshminarayana, A. Raghunathan, K. S. Khouri, N. K. Jha, and S. Dey, "Common-Case Computation: A High-Level Technique for Power and Performance Optimization," Proc. of DAC, pp 56-61, 1999.

[10] Y. Luo, J. Yu, J. Yang, and L. Bhuyan, "Low Power Network Processor Design Using Clock Gating," Proc. of DAC, pp. 13-17, 2005.

[11] H. M. Jacobson, "Improved Clock-Gating through Transparent Pipelining,” Proc. of ISLPED, pp. 26-31, 2004.

[12] N. Banerjee, K. Roy, H. Mahmoodi, and S. Bhunia, "Low Power Synthesis of Dynamic Logic Circuits Using Fine-Grained Clock Gating," Proc. of DATE, pp. 6-10, 2006.

[13] A. P. Hurst, "Automatic Synthesis of Clock Gating Logic with Controlled Netlist Perturbation," Proc. of DAC, pp. 654-657, 2008 